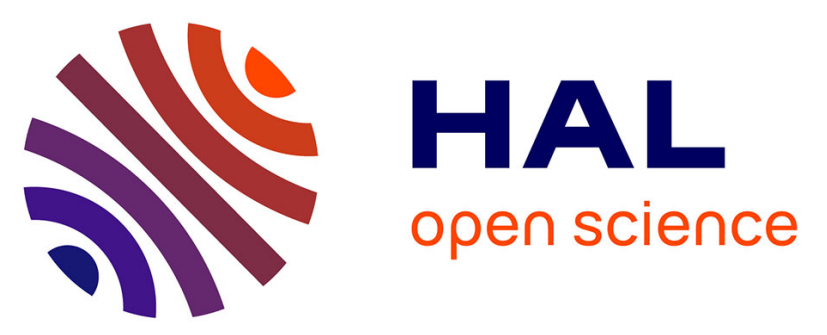

\title{
TWO GENERALISED THEORIES DESCRIBING LIGHT DIFFRACTION BY ULTRASONIC PULSES :A. GENERATING-FUNCTION METHODB. LAPLACE-TRANSFORM METHOD
}

\author{
K. van den Abeele, O. Leroy
}

\section{To cite this version:}

K. van den Abeele, O. Leroy. TWO GENERALISED THEORIES DESCRIBING LIGHT DIFFRACTION BY ULTRASONIC PULSES:A. GENERATING-FUNCTION METHODB. LAPLACETRANSFORM METHOD. Journal de Physique Colloques, 1990, 51 (C2), pp.C2-1235-C2-1238. 10.1051/jphyscol:19902290 . jpa-00230624

HAL Id: jpa-00230624 https://hal.science/jpa-00230624

Submitted on 1 Jan 1990

HAL is a multi-disciplinary open access archive for the deposit and dissemination of scientific research documents, whether they are published or not. The documents may come from teaching and research institutions in France or abroad, or from public or private research centers.
L'archive ouverte pluridisciplinaire HAL, est destinée au dépôt et à la diffusion de documents scientifiques de niveau recherche, publiés ou non, émanant des établissements d'enseignement et de recherche français ou étrangers, des laboratoires publics ou privés. 
COLLOQUE DE PHYSIQUE

Colloque C2, supplément au $\mathrm{n}^{\circ} 2$, Tome 51, Février 1990

ler Congrès Français d'Acoustique 1990

TWO GENERALISED THEORIES DESCRIBING LIGHT DIFFRACTTON BY ULTRASONIC PULSES :

A. GENERATING-FUNCTION METHOD

B. LAPLACE-TRANSFORM METHOD

\author{
K. VAN DEN ABEELE $(1)$ and O. LEROY \\ Interdisciplinar Research Center, K.U.L. Campus Kortrijk, B-8500 \\ Kortrijk, Belgium
}

RDSUME: Commençant par les équations de Maxwell, nous dérivons deux modèles théoriques qui décrivent la diffraction de la lumière par des pulsations ultrasoniques. Dans le premier modèle, basé sur une fonction génératrice, nous trouvons des solutions analytiques pour les amplitudes et les intensitées des ondes diffractées, "qui sont seulement valable dans le régime de Raman-Nath. Le second modèle est décrit par une méthode numérique, utilisant les transformations de Laplace. Ce modèle décrit aussi bien le régime de Raman-Nath que le régime de Brags. Les deux méthodes sont comparées aver une théorie existante basée sur l'intégral de diffraction. Nous concluons que nous retrouvons ces derniers résultats considérant le régime extrème de Raman-Nath $p=0$ dans nos modèles. Une règle générale concernant la propriété de $\mathrm{l}^{\prime}(\mathrm{a})$ symétrie du spectre, en incidence normale, est dérivée des équations générales de Raman-Nath, utilisant la méthode de la fonction génératrice.

ABSTRACT: As an extension from previous studies concerning the interaction of light with ultrasound of a purely sinusoidal form, two theoretical models describing light diffraction by ultrasonic pulses are derived starting from the Maxwell equations. Based on a generating function, the first model provides finite solutions for the amplitudes and intensities of the diffaction pattern and is restricted in validity to the Raman-Nath region. The second model consists of a numerical method developed by means of Laplacetransformations and covers as well the Raman-Nath as the Bratgs region. Both new methods are compared with a theory based on the diffraction-integral theory, leading to the conclusion that the results of this latter method can be obtained from our models considering the extreme Raman-Nath regime $\rho=0$. A general rule concerning the (a)symmetry properties of the diffraction spectrum at normal incidence has been derived from the extended Raman-Nath equations, using the generating function method.

\title{
1. INTRODUCTION :
}

Recent developments in Non-Destructive Testing and medical diagnosis oncouraged more and more physicists to study pulsed ultrasound instead of continuous plane waves. Our current interest is to describe the interaction of plane wave laser light with ultrasonic pulses at normal incidence. Extending known theories for continuous supersonic waves $[1,2]$ to pulses, we calculate the amplitudes and intensities of the diffracted light in the fraunhofer pattern in two different manners. Both models start from the Maxwell equations and follow the light through a medium with a refractive index which is modulated due to the ultrasonic pressure of the pulse. The time-history of this refractive index can be expressed by a fourier sine expansion which proves that a pulse can be identified with a superposed wave :

$$
\mu(x, t)=\mu_{0}+\mu \sum_{j=1}^{\infty} \alpha_{j} \sin \left(2 \pi j\left[\nu_{p}^{*} t-\frac{x}{\lambda_{p}^{*}}\right]+\delta_{j}\right)
$$

where

$$
\begin{aligned}
& \nu_{\mathrm{p}}^{*}=\text { repetition frequency } \\
& \mu_{0}=\text { refractive index of the undisturbed medium } \quad \lambda_{\mathrm{p}}^{*}=\text { length of the pulse in the medium } \\
& \mu \cdot \alpha_{j} \text { and } \delta_{\mathrm{j}} \text { are the amplitude and phase of the } \mathrm{j} \text {-th Fourier-component of the pulse. }
\end{aligned}
$$

Considering Maxwell's equations as a starting point, Leroy [3] derived that the amplitudes of the diffraction orders in the farfield satisfy the extended Raman-Nath equations :

$$
\begin{aligned}
& 2 \frac{d \Psi_{n}}{d \zeta}(\zeta)-\sum_{j=1}^{n} a_{j}\left[\Psi_{n-j}(\zeta) \exp \left(-i \delta_{j}\right)-\Psi_{n+j}(\zeta) \exp \left(i \delta_{j}\right)\right]=i p n^{2} \Psi_{n}(\zeta) \\
& \text { with boundary conditions } \Psi_{n}(\zeta=0)=\delta_{n, 0} \quad(n:-\infty \ldots+\infty) \\
& \text { where } \zeta=\frac{2 \pi}{\lambda} \mu \mathrm{z} \quad \text { and } \quad \rho=\frac{\lambda^{2}}{\mu_{0} \mu \lambda_{\mathrm{p}}{ }^{2}}
\end{aligned}
$$

The solution $\Psi_{n}(\zeta)$ is the amplitude of the diffracted lightbeam whose direction with regard to the incident laser-beam is defined by the angle $\theta_{n}=-\arcsin \left(\frac{n \lambda}{\lambda_{p}^{*}}\right)$ while its frequency will be $\nu-n \nu_{p}^{*}$ and the intensity is given by

$$
I_{-n}(\zeta)=\Psi_{n}(\sigma) \cdot \Psi_{n}^{*}(\zeta)
$$

We now propose two methods to integrate the systern of difference-differential equations (2).

(1) Aspirant of the National Foundation for Scientific Research of Belgium. 

GENER ATING FUNCTION METHOD.

Considering $\Psi_{n}(\zeta)$ to be the coefficients of the Laurent expansion of an unimown generating function $G(\zeta, \eta)$, which we suppose to be holomorphic in an annular region with centre $O$ in the complex $\eta$ plane $\left(G(\zeta, \eta)=\left.\sum_{n=-\infty}^{\infty}\right|_{n}(\zeta) \eta^{n}(4)\right)$ we find that $G(\zeta, \eta)$ satisfies the following equation

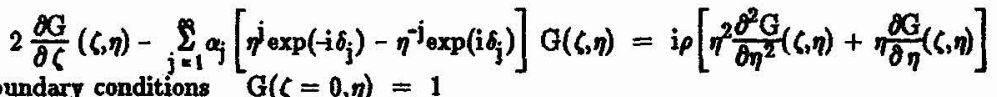

with boundary conditions $G(\zeta=0, \eta)=1$

Once the function $G(\zeta, \eta)$ is known, the amplitudes of the diffracted light waves may be found by expanding $G(\zeta, \eta)$ into a Laurent-series.

In order to integrate (5) we propose a solution in the form of a series in $p: G(\zeta, \eta)=\sum_{k=0}(i p)^{k} G_{k}(\zeta, \eta)$

Substituting this expansion into (5) and we obtain a partial differential equation for each integer value of $n \geq 0$ with the appropriate boundary condition. If we assume that $p$ is only a small value, we neglect in (6) terms of $p^{2}$ and higher. BJ solving these equations for $n=0$ to 2 , we have found an approximate solution for $G(\zeta, \eta)$ :

$$
G(\zeta, \eta)=G_{0}(\zeta, \eta)+i p G_{1}(\zeta, \eta)-\rho^{2} G_{2}(\zeta, \eta)
$$

Comparing the coefficients of $\eta^{\mathrm{n}}$ in (4) and (7), we get an analytical expression for the diffracted lightwave of order $n$ in the form of $\Psi_{n}(\zeta)=\Psi_{n}^{(0)}(\zeta)+i \rho \Psi_{n}^{(1)}(\zeta)-\rho^{2} \Psi_{n}^{(2)}(\zeta)$

An interesting remart however is that $\Psi_{n}^{(0)}$ corresponds to the amplitude of the $n$-th diffraction order in the case of extreme Raman-Nath regime $(\rho=0)$ and is exactly the same expression as Neighbors and Majer [4] found by means of the diffraction-integral theory. As $\Psi_{n}^{(0)}$ is only the first term in our expression for the diffracted amplitudes, we can consider i $\rho \Psi_{n}^{(1)}$ and $-\rho^{2} \Psi_{n}^{(2)}$ as corrections to the theory of Neighbors and Mayer (see Fig 1.a and b.)
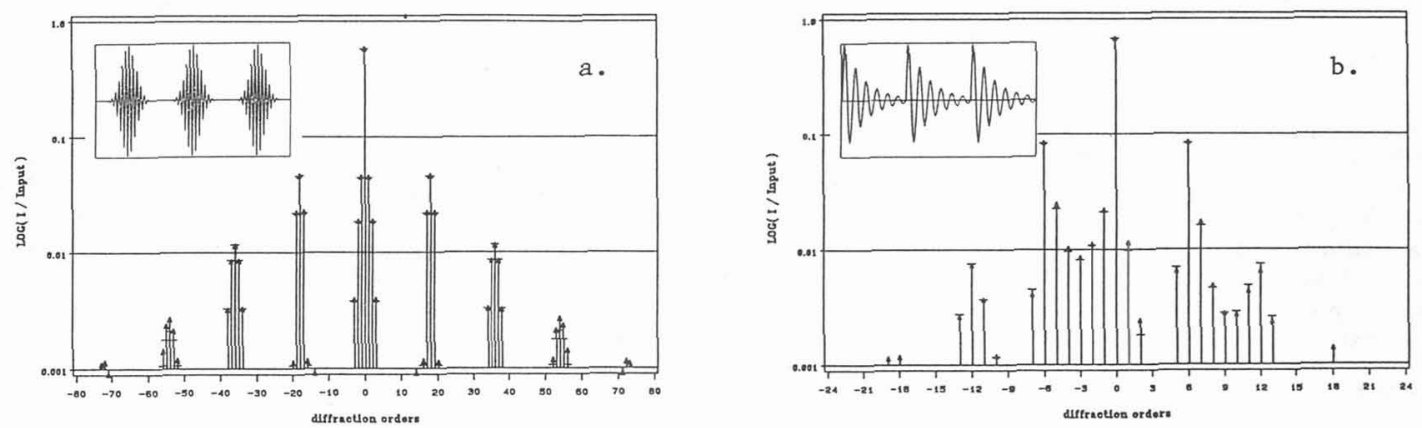

Fig 1. a. Farfield diffraction pattern of normally incident light on a gasssian shaped ultrasonic palse (window). Results of the generating function method for $p=0.0(-)$ and $\rho=9.0 E-04(\Delta) \quad(v=2.5)$

b. Farfield diffraction patiem of normally incident light on an exponentially damped uhrasonic pulse (window). Resslts of the generating function method for $\rho=0.0(-)$ and $\rho=1.1 E-0.8(\Delta)(v=2.0)$

For a purely sinusoidal ultrasonic wave, the formulae obtained by Kuliasko et al [1] are exactly reproduced. 3. CALCULATION OF THE FARFIELD AMPLITUDES OF THE DIFFRACTION PATTERN BY MEANS OF A
LAPLACE-TRANSFORM METHOD.
Defining $\mathbf{k}_{1}=0 / \nu_{\mathrm{p}}^{*}\left(\nu_{0}^{*}=\right.$ the centre modulation frequency, $\nu_{\mathrm{p}}^{*}=$ the repetition frequency $)$ and $\beta=$ arcsin $\left[\frac{\lambda}{2 \lambda_{p}^{*}}\right]$, it is
known from theoretical and experimental studies $[4-7]$ that the primary orders in the fraunhofer diffraction pattern show up in the directions $k_{1}+2 \beta$ and at most $k_{1}-1$ secondary orders can be observed between two primary orders at every even multiple of the angle $\beta$. Also from previous studies we know that, in the case of normal incidence of the laser beam, as many positive as negative primary diffraction orders will contribute to the final spectrum, while the number of secondary orders left and right of a peak can differ for one primary order compared to another.Suppose now that only $2 N+1$ primary orders (N positive and $N$ negative) have a non-zero contribution of intensity to the final diffraction spectrum and that for the $k$-th primary order ( $-N \leq$ $k \leq N)$ only $P(k)$ secondary orders to the left and $Q(k)$ secondary orders to the right of this peak occur. If we assume that all other amplitudes are vanishing small, the infinite system (2) is reduced to a finite set of equations.Applying the Laplace-transform to the truncated system and substituting

$$
\left.\phi_{n}(s)=\mathcal{L} \Psi_{n}(\zeta)\right)=\int^{\infty} \Psi_{n}(\zeta) e^{-s \zeta_{d} \zeta}
$$

we obtain an algebraic system of equations which can be written in matrix notation as follows : 
$[\mathrm{D}-\mathrm{t} \mathrm{t}] \phi(\mathrm{t})=2 \mathrm{i} \mathrm{E}$

where $I$ is the unit-matrix of dimonsion $d$ with $d=\sum_{j=-N}^{N}[P(k)+Q(k)+1] \quad$ and $D$ a hermitain matix.

$\phi(t)=\phi\left(\frac{i t}{2}\right)$ with $\phi(s)$ given by the collumn matrix :

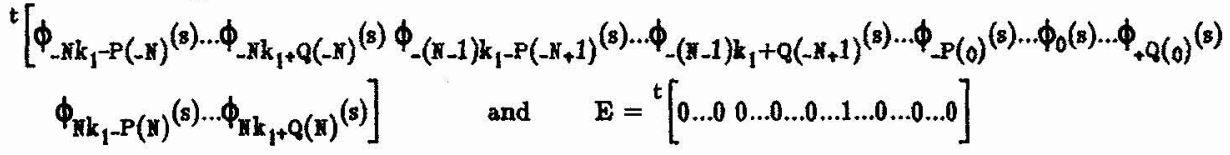

The unknown Laplace transformed functions $\phi_{n}(t)$ can be calculated from $(9)$ using Cramer's method, and applying the Heaviside expansion theorem [8] for the special case in which $D$ has only single eigenvalues we get :

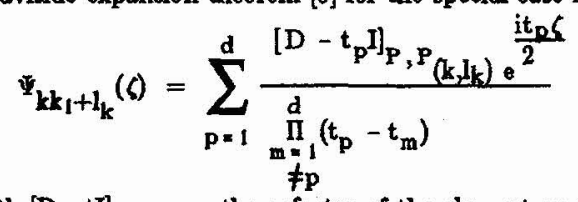

with $[D-t I]_{P, P}\left(k, l_{k}\right)$ the cofactor of the element on the position $\left(P, P\left(k, l_{k}\right)\right.$ in $(D-t I), t_{p}$ an eigenvalue of $D$ and $P=\sum_{j=-N}^{-1}[P(j)+Q(j)+1]+P(0)+1 \quad P_{\left(k, l_{k}\right)}=\sum_{j=-1}^{k-1}[P(j)+Q(j)+1]+1_{k}+P(k)+1$

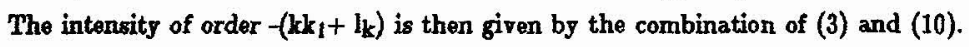
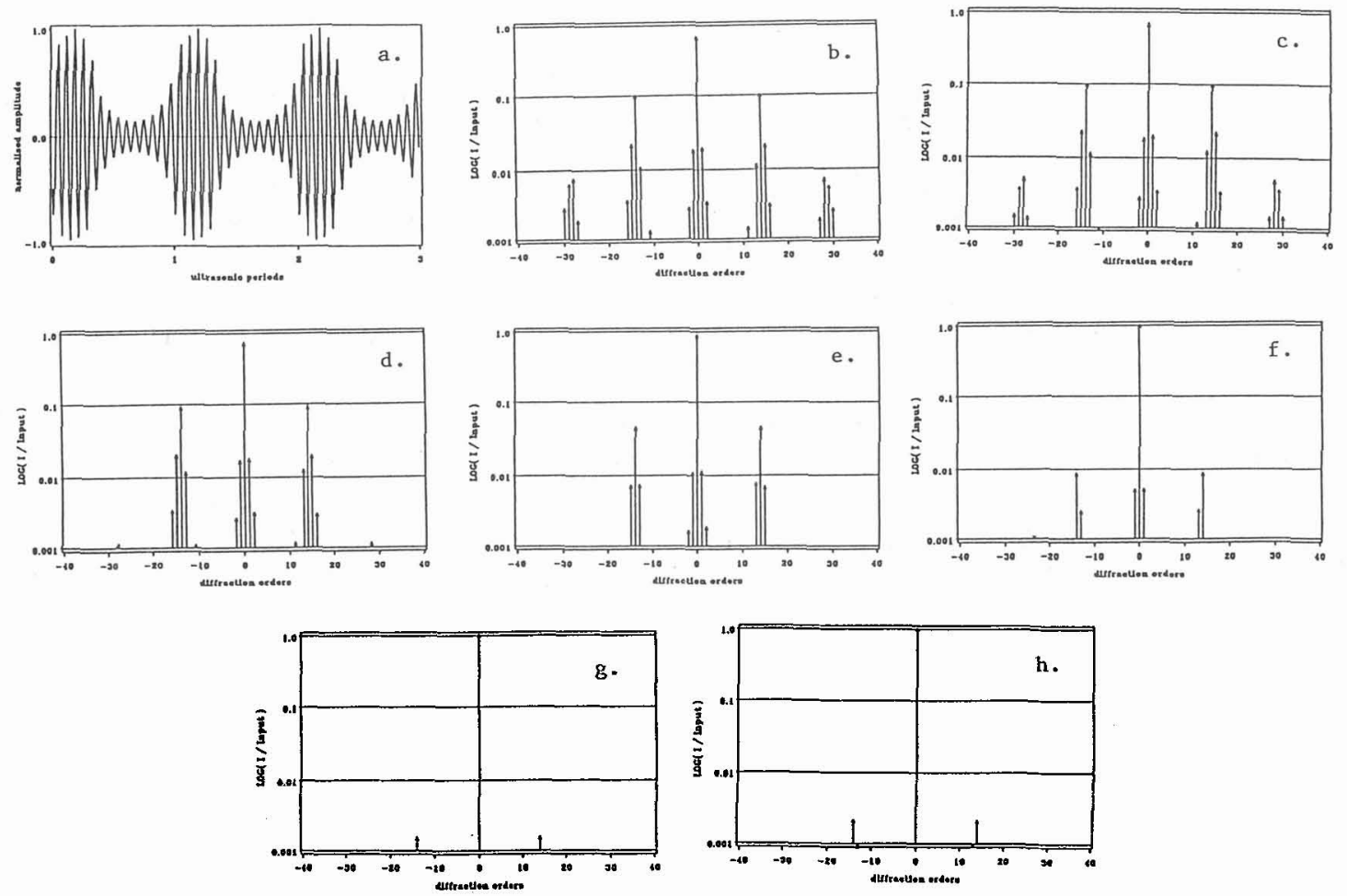

Fig 8. : Dependence of the farfield diffraction pattern on p. The diffracting pulse is thown in fig. 2a. (from J. Walf [7])

2b. $\rho=1.0 E-07$ or $\rho O=2.19 E-05$

2d. $p=1.0 E-02$ or $p O=2.19$

2). $p=3.0 E-02$ or $p 0=6.57$

क. $p=9.0 E-08$ or $p O=19.71$

2c. $p=5.0 E-03$ or $P O=1.09$

2e. $\beta=2.0 E-0.8$ or $\rho 0=4.38$

2f. $p=7.0 \mathrm{~B}-02$ or $p O=15.39$

Remark that for this numerical method, no restrictions on the Raman-Nath parameters $\rho$ and $v$ are assumed beforehand, so that, by choosing $N$ and $\{(P(k), Q(k)) \mid k ;-N$...N\} in an appropriate way, this model can cover as well the Raman-Nath as the Brage region. The influence of $p$ on the diffraction pattern caused by interaction of light with the ultrasonic pulse ahown in 
fig $2 \mathrm{a}$ is illustrated in fig $2 \mathrm{~b}-2 \mathrm{~h}$. Important changes in the pattern only occur when $\rho_{0}$ takes on values higher than 1 ( $\rho_{0}=$ $\frac{\lambda^{2}}{H_{0} f \lambda_{0}^{2}}$, $\lambda_{\delta}^{*}$ the centre modulation wavelenght of the pulse ) : the number of primary orders diminishes, satallite lines vanish and when $\rho$ is large enough only zeroth, first and minus first primary orders are measurable.

At last, we visualise the conversence of our new Laplacetransformtheory towards the MN-OA-method of $\mathrm{E}$. Blomme and $\mathrm{O}$. Leroy [2] which was derived for continuous waves. Increasing the variance-parameter of a gaussian-shaped pulse, the diffraction pattern changes in this way that satellite lines lose part of their intensity while this lost of energy is compensated by an increase of the intensity of the primary onders (except for the seroth onder). When the variance-parameter is lange enough, the farfield spectrum is exactly the same as the one obtained by the MN-OA-method for normal incidence of light on a continuous ultrasonic wave : no satelitite lines and a perfect symmetric pattern.
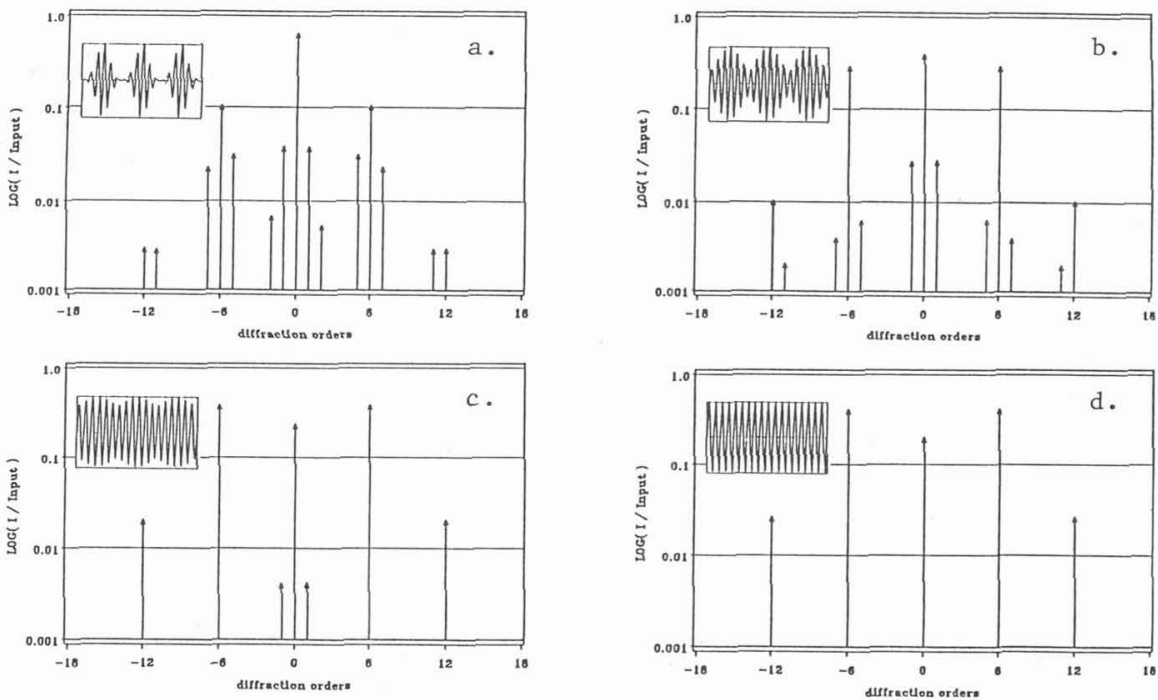

Fig 3. : Convergence of the diffraction patterns of gaussian shaped pulses $\left(k_{1}=6\right)$ calculated by means of the Laplace-transform method, rowards the continuous wave limit (same results as the $M N-O A-\operatorname{method}[2]$ ). $(v=2.0, p=4.166 E-02$ or $p 0=1.5)$

\section{GENERAL THEORY CONCERNING SYMMETRY-PROPERTIES OF THE DIFFRACTION PATTERN}

Replacing the complex variable $\eta$ by $\exp (\mathrm{iA}) \eta^{-1}$ (A being any real number) in (4) we find that

$\tilde{G}(\zeta, \eta)=\sum_{n=-\infty} \Psi_{-n}(\zeta) \exp ($ inA $) \eta^{n}$ satisfies the same partial differential equation and boundary condition as $G(\zeta, \eta)$ provided

that: $A=2 \delta_{1}+(2 k-1) \pi \quad$ and $\quad \delta_{j}=j \delta_{1}+[(21-1)-j] \frac{\pi}{2} \quad(k, 1 \in \mathbb{Z})$

As the solution is unique, we may conclude that under these restrictions $\Psi_{n}(\zeta)=\Psi_{-n}(\zeta) \exp ($ in $A)$ so that the diffraction pattern is symmetric with respect to the zeroth order. This condition, which is rather strong, is generally not fulfilled in the case of pulses, so that mostly the diffraction pattern will be assymmetric.

\section{CONCLUSION}

The two new methods derived from the Maxwell equations demonstrate in a very satisfying way the splitting of the diffraction pattern into primary and secondary orders and the property of asymmetry of the spectrum in the case of normal incidence of plane wave laser light on ultrasonic pulses. Both models gain an advantage in accuracy over the diffraction-integral theory used in previous studies. Especially the Laplace-transform method is a very powerfull mothod which is valid as well in the Raman-Nath as in the Bragg region.

\section{REFERENCES}

11/ KULIASKO F., MERTENS R. and LEROY O., Proc. Indian Acad. Sci., 67, 295-302, 1968.

/2/ BLOMME E. and LEROY O., Acustica, 63; 83-89, 1987.

3/ LEROY O., Ultrasonics, 10, 182-186, 1972.

14/ NEIGHBORS T.H. and MAYER W.G., J. Acoust. Soc. Am. 74, 146-152, 1983.

/5/ HAUSLER E., MAYER W.G. and SCHWARTZ M., Acoustics Letters Vol 4, 9, 180-194, 1981

/6/ MAYER W.G. and NEIGHBORS T.H, 10 th International Symposium on Nonlinear Acoustics, 113-116, 1984.

/7) WOLF J., NEIGHBORS T.H. and MAYER W.G., Ultrasonics, 27, 150-154, 1989.

/8/ DAVIS B., Springer-Verlag, Part I , \$6, 1978. 